\title{
Search of critically important objects of the gas industry with the method of determining critical elements in networks of technical infrastructures
}

\author{
Sergey Vorobev $^{1 *}$, Alexey delev $^{1}$, Elena Smirnova $^{1}$ \\ ${ }^{1}$ Melentiev Energy Systems Institute, 130 Lermontov str., Irkutsk, Russia
}

\begin{abstract}
The paper shows the application of the method of determining critical elements in the networks of technical infrastructures in the search and analysis of the importance of critical gas industry facilities. The purpose of this method is to search for critical elements and sets of elements in network infrastructures and their ranking by importance. The paper presents an adaptation of the method for determining critical elements in networks of technical infrastructures for the model of gas supply system functioning, which consists in describing the response of the gas supply system to the failure of a single element or a group of them. Conclusions are made about the expediency of using this method in research into the search for critical gas industry objects and determining their significance.
\end{abstract}

\section{Introduction}

One of the main aspects of ensuring energy security is to create the conditions for the greatest possible degree of satisfaction of consumers with energy resources in emergency situations. Investigation of this aspect of energy security requires the definition of critical objects for the fuel and energy complex in general and for individual energy systems. It means the definition of those facilities, partial or complete failure of which can cause significant damage to the country from the fuel and energy sector.

The need to define and analyse the critical objects in the fuel and energy sector is also dictated by the fact that the functioning of the fuel and energy complex and its constituent cells is the most important factor in ensuring the normal vital activity of the economy of the whole country and its individual regions.

Abroad, there are many studies devoted to the study of the vulnerability of certain critical infrastructures in the event of an accidental disturbance, a deliberate attack or a natural disaster. In the case of insufficient knowledge of the infrastructure, probabilistic methods of risk analysis are used to assess its vulnerability $[1,2]$. If historical data are stored, then the theory of statistics is used to analyze and predict the impact of natural disasters on the performance of infrastructure [3]. To take into account the topology of the infrastructure, when analyzing its structural vulnerability, network approaches are used, for example, the theory of complex networks [4]. Recently, in foreign studies, more attention has been paid to the consideration of interconnected infrastructures [5] and the impact of interaction between them on their vulnerability $[6,7]$.
In this paper we will talk about the gas industry. The matter is that today more than $90 \%$ of Russian natural gas is extracted in one gas producing region (NadymPur-Tazovsky district of the Tyumen region). This region is located in 2-2.5 thousand $\mathrm{km}$ from the main gas consumption areas and in 4-5 thousand $\mathrm{km}$ from the countries that import this gas. Thus, all Russian gas is transported over long distances through the system of main gas pipelines, which have a large number of crossings and bridges, moreover, the threads of powerful gas mains are often laid at a small distance from each other. At present, in the gas transportation system of Russia, it is possible to note more than 20 crosses of main gas pipelines that potentially dangerous for system. Previously, a number of studies devoted to the identification of critical objects in the gas industry were carried out [8]. The list of crossings of main gas pipelines in the Unified Gas Supply System of Russia has been determined, the violation of which will lead to a relative deficit of gas supplies for the system as a whole of $5 \%$ or more.

Also, conducted studies have shown that along with main gas pipelines crossings, a significant shortage of gas supplies through the system ( $5 \%$ or more) may result in a disruption of the functioning of both a separate section of the main gas pipelines located between the node compressor stations and the disruption of several such sites. The determination of such sections of main gas pipelines and their combinations is complicated by the fact that in the current configuration of the Unified Gas Supply System of Russia there are about 500 edges. Therefore, at this stage of the study of critical objects of the gas industry, the authors proposed the use of the method of determining critical elements in the networks 
of technical infrastructures [9]. Its application is justified by the need to assess the importance of the gas sector's critical objects.

\section{Definition and ranking of critical elements}

The definition of critical elements, as a rule, is a simple task when considering only single failures. When considering several simultaneous failures, this task can become much more complicated.

It is especially difficult to determine the critical groups of elements with the synergistic effect. In this context, the synergistic effect means that the negative consequences of the group's failure are wholly higher than the total impact of individual failures of the elements within the group. In other words, the failure of a group of two elements with serious negative consequences can give a synergistic effect if the failure of each of the elements in itself is not the cause of any significant consequences.

The method for determining critical elements in technical infrastructure networks [9] facilitates the definition and ranking of such groups of elements (as well as groups of elements whose failure does not give a synergistic effect). The found critical elements or sets of elements can then be studied in more detail using probabilistic methods of risk analysis [10].

The essence of the method is to investigate the set of failures, each of which represents a set of faulty elements, has only one negative effect on the system is characterized by a size that specifies the number of elements whose failure occurs simultaneously.

The size of the set of failures $n$ is chosen by the researcher depending on the total number of elements of the system $t$. However, based on practical considerations, $\mathrm{n}$ should not exceed 3 or 4 , since the number of possible failure sets, equal to $t ! /((t-n) ! * n !)$, Grows rapidly with increasing $n$. The ranking of failure sets is carried out in accordance with the magnitude of their synergistic effects.

If the set of failures $F$ consists of $n$ elements $c_{l}, \ldots, c_{n}$, $n>1$, then $F=\left\{c_{1}, \ldots, c_{n}\right\}$. The elements of the set of failures can be divided into subsets of $S$. This partition can be performed in several ways. Let $V_{i}$ denote the set containing the subsets of $S$ for the particular partition method $F$ and $P$ denote the number of partition methods. The concrete subset belonging to $V_{i}$ is denoted by $S_{i j}$. If we denote the number of such subsets of $m$, then $V_{i}$ will consist of the series $S_{i l}, \ldots, S_{i m}$. Since the subsets are constructed on the basis of the breakdown of $S$, then all the elements contained in the subsets also belong to the set of failures and each element can be contained in only one subset for each partitioning method.

A lot of failures have a synergistic effect only if the negative consequence of failures $C(F)$ is greater than the sum of the consequences for subsets of the set $F$ for all possible partitions $V_{l}, \ldots, V_{p}$.

$$
C(F)>\sum_{j=1}^{m} C\left(S_{j}^{i}\right) \forall V_{i}:
$$

$$
\begin{gathered}
F=\left\{c_{1}, \ldots, c_{n}\right\}, n>1, \\
S_{j}^{i} \subset F, S_{1}^{i} \cap \ldots \cap S_{m}^{i}=\varnothing, S_{1}^{i} \cup \ldots \cup S_{m}^{i}=F, j=1, \ldots, m \\
V_{i}=\left\{S_{1}^{i}, \ldots, S_{m}^{i}\right\}, i=1, \ldots, p .
\end{gathered}
$$

The synergistic effect of the set of failures $C_{s y n}(F)$ is defined as the difference between the consequences due to the set of failures and the largest sum of consequences from subsets for all possible partitions of $V$.

$$
C_{s y n}(F)=C(F)-\max _{V_{i}}\left(\sum_{j=1}^{m} C\left(S_{j}^{i}\right)\right)
$$

The synergistic effect cannot be calculated on the basis of the consequences of the individual considered failures, since it manifests only when all elements of the set are simultaneously rejected, that is, it is a consequence additional to the effect of subsets of failures. For example, the synergistic effect of a set of failures of size 3 sets cannot be calculated by summing the consequences of its subsets of size 2 and 1 . Thus, such critical sets of failures cannot be determined only by considering combinations of elements that are critical in themselves.

When considering the issue of two simultaneous failures, the criticality of a particular element is considered as a vulnerability of the system to failures of this element and one of the other elements. There are a large number of sets of failures of size 2, which include this element, and every set of failures is associated with a certain consequence. Thus, the vulnerability of a system can be described with a set of failure sets, including a description of the consequences of failure of each set of failures. Vulnerability indicators that help to compare the criticality of different elements can be obtained by analysing a set of failure sets. One such method is the averaging of the consequences of all sets of failures that contain a certain element. The obtained value can be interpreted as the average value of the consequences due to failures of a particular element and another element selected randomly (for failure sets of size 2).

The indicator by which the elements making the main contribution to the synergistic effect of a certain set of failures can be determined in [2] is calculated as follows

$$
\operatorname{Con}_{\text {sizen } n}\left(c_{i}\right)=\frac{\sum C_{\text {syn }}\left(F \mid c_{i} \in F, n\right)}{\sum C_{\text {syn }}(F \mid n)}
$$

$\sum C_{s y n}\left(F \mid c_{i} \in F, n\right)$

then the sum of the synergistic effects of all sets of failures of size $n$ that contain the element $c_{i}$.

$\sum C_{s y n}(F \mid n)$ the sum of the synergistic effects of all sets of failures of size $n$. The indicator (3) expresses the contribution of synergetic consequences from a particular element to the aggregate synergistic effect of a set of failures of a certain size. Thus, the exponent (3) will be high for such an element $c_{i}$, which is contained in 
a large number of sets of failures with a significant synergistic effect.

\section{Determination of critical elements in the energy system}

Essential aid in the research of the critical elements in the gas industry can be provided by geoinformation systems (GIS) as a means of visualizing information describing complex spatially-distributed systems, such as energy systems. The basic principles of mapping energy system schemes and searching for weak places were developed in the process of creating and using the problem-oriented GIS "Oil and Gas of Russia" for studying the survivability of individual energy systems, in particular, gas supply systems, oil and oil products systems [11-13]. The scheme of the energy system in the GIS is presented in the form of a map consisting of two layers with vector data. The first layer, represented by point objects, characterizes the condition of the producers, and also shows whether the needs of energy consumers are completely satisfied. The second layer, represented by lines, gives an idea of the degree of congestion of the transport subsystem. Attributive information on the production and consumption of energy resources is associated with the point objects of the first layer, and information on the sections of the main pipelines (throughput, number of pipes, their diameter, etc.) connects with the lines of the second layer.

To assess the importance of the critical elements in the Unified Gas Supply System of Russia using the method of determining critical elements in technical infrastructure networks, it is necessary to adapt the latter to the functioning model of the energy system. This adaptation consists in describing the reaction of the energy system to the failure of a single element or a group of them. The adaptation of the method for assessing the significance of the critical elements in the Unified Gas Supply System of Russia is shown below.

The flow distribution model in the Unified Gas Supply System of Russia in the "Oil and Gas of Russia" is designed to assess the production capabilities of the Unified Gas Supply System of Russia in conditions of various kinds of disturbances. The purpose of such studies is to minimize gas deficits at the consumption sites. The Unified Gas Supply System of Russia in the model is represented as a set of three subsystems: gas sources, main gas transport network and consumers.

When solving the problem of estimating the state of a system after a perturbation, the criterion of the optimality of the distribution of flows is the minimum gas deficit in the consumer with minimum costs for delivering gas to consumers. This problem can be solved by finding the maximum flow over the network. The problem of the maximal flow was investigated in [14] and is formulated as follows:

with follow conditions $f \rightarrow \max$,

$$
\begin{aligned}
& \sum_{i \in N_{j}^{+}} x_{i j}-\sum_{i \in N_{j}^{-}} x_{j i}=\left\{\begin{array}{c}
-f, j=O \\
0, j \neq O, S \\
f, j=S
\end{array}\right. \\
& 0 \leq x_{i j} \leq d_{i j}, \text { for all of }(\mathrm{i}, \mathrm{j}) .
\end{aligned}
$$

In this formulation, $f$ is the maximized variable corresponding to the maximum flow.

With complex schemes, which are the Russian and European gas transmission networks, there may be several solutions, that is, several possible maximum flows. Then it is advisable to talk about minimizing the cost of delivering gas to consumers and using the Basaker-Gowen algorithm [14]:

$$
\begin{aligned}
& \sum_{(i, j)} C_{i j} x_{i j} \rightarrow \min , \\
& x \in X^{s} .
\end{aligned}
$$

Its application allows us to determine the maximum flow of the resource at its minimum cost or the optimal volumes of daily gas from the underground gas storages, which maximally provide the given volumes of gas supply to consumers at the minimum costs for extraction, transportation of gas and its withdrawal from underground gas storages.

$O$ - the node number of the common source; $S$ - is the number of the common drain node; $N_{j}^{+}$- is a subset of edges entering into the node $j ; N_{j}^{-}$is the subset of outgoing edges from the node $j ; f-$ is the value of the total flow over the network; $x_{i j}$ - is the flow along the edge $(i, j) ; d_{i j}$ - restrictions on the flow along the edge $(i$, $j) ; X^{*}$ - is the set of solutions of problem (4)-(6); $C_{i j}-$ specific costs for gas transportation.

The node $O$ is connected by fictitious edges with all real sources of energy resources, and node $S$ - with all consumers.

Similarly to [1], the total shortage of energy resources to consumers is used to estimate the impact of multiple failures $\mathrm{F}$ :

$$
C(F)=\sum_{i \in N_{S}^{+}} d_{i S}-f
$$

$N_{S}^{+}$- is a subset of the edges that "enter" the common $\operatorname{sink} S$,

$d_{i S}$ - gas demand in the country and deliveries for export. The synergistic effect of the failure set $F$ is calculated according to (2).

\section{Assessment of importance of critical elements of the gas industry}

The scheme of the Unified Gas Supply System of Russia used for the calculations in this paper on the flow model takes into account all the main features of the operation of the Unified Gas Supply System of Russia and contains:

- 382 nodes, including:

- 28 gas sources;

- 64 gas consumers (subjects of the Russian Federation); 
- 24 underground gas storage facilities;

- 266 nodal compressor stations;

- 486 edges representing main gas pipelines and outlets to distribution gas networks.

On this scheme of the Unified Gas Supply System of Russia several calculations were made to determine the criticality of individual edges in the gas transmission network:

1 - the operation of each edge of the scheme was interrupted in turn, and the total daily gas deficit was determined by the system (million $\mathrm{m} 3$ per day) as a result of such a violation;

2 - the functioning of a system with multiple failures $\mathrm{n}=2$;

3 - the functioning of a system with multiple failures $\mathrm{n}=3$.

Baseline data for calculations, such as daily gas production, consumption, export and import volumes, throughput capacity of existing gas pipelines, are taken in accordance with official statistical information [15-17] for 2016 .

Table 1. Results of determining the criticality of individual edges in the gas transmission network

\begin{tabular}{|c|c|c|c|c|}
\hline \multirow{2}{*}{ 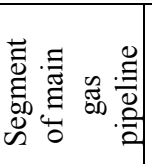 } & \multirow{2}{*}{$\begin{array}{c}\text { Gas } \\
\text { deficit, } \\
\text { mln. } \\
\mathrm{m}^{3} / \text { day. }\end{array}$} & \multirow{2}{*}{$\begin{array}{l}\text { Total } \\
\text { gas } \\
\text { deficit, } \\
\%\end{array}$} & \multicolumn{2}{|c|}{$\begin{array}{l}\text { The value of the } \\
\text { criterion of the object } \\
\text { when }\end{array}$} \\
\hline & & & $\mathrm{n}=2$ & $\mathrm{n}=3$ \\
\hline A & 412,70 & 21 & 0,0739 & 0,0756 \\
\hline B & 412,70 & 21 & 0,0752 & 0,0784 \\
\hline $\mathrm{C}$ & 412,70 & 21 & 0,0763 & 0,0807 \\
\hline $\mathrm{D}$ & 295,01 & 15 & 0,0573 & 0,0614 \\
\hline $\mathrm{E}$ & 295,01 & 15 & 0,0573 & 0,0614 \\
\hline $\mathrm{F}$ & 175,54 & 9 & 0,0352 & 0,0424 \\
\hline G & 174,75 & 9 & 0,0351 & 0,0423 \\
\hline $\mathrm{H}$ & 160,68 & 8 & 0,0327 & 0,0401 \\
\hline I & 156,40 & 8 & 0,0354 & 0,0418 \\
\hline $\mathrm{J}$ & 156,40 & 8 & 0,0369 & 0,0444 \\
\hline K & 152,54 & 8 & 0,0313 & 0,0386 \\
\hline $\mathrm{L}$ & 112,24 & 6 & 0,0218 & 0,0263 \\
\hline $\mathrm{M}$ & 92,70 & 5 & 0,0237 & 0,0318 \\
\hline $\mathrm{N}$ & 82,70 & 4 & 0,0255 & 0,0348 \\
\hline $\mathrm{O}$ & 79,20 & 4 & 0,0303 & 0,0411 \\
\hline $\mathrm{P}$ & 78,72 & 4 & 0,0302 & 0,0410 \\
\hline $\mathrm{Q}$ & 74,71 & 4 & 0,0161 & 0,0211 \\
\hline $\mathrm{R}$ & 74,71 & 4 & 0,0161 & 0,0211 \\
\hline $\mathrm{S}$ & 51,60 & 3 & 0,0226 & 0,0325 \\
\hline $\mathrm{T}$ & 51,60 & 3 & 0,0227 & 0,0324 \\
\hline
\end{tabular}

Table 1 clearly demonstrates that the first 5 segments of main gas pipelines "A" - "E" are critical both in terms of the maximum gas deficit in consumers and in their contribution to the synergistic effect that is possible with simultaneous disruption of the functioning of several segments of main gas pipelines. Violation of the functioning of these segments will lead to the emergence of a significant gas deficit in consumers, from 15 to $21 \%$ in total across the gas transmission network. All these factors make it possible to classify the segments of main gas pipelines "A" - "E" to critical objects of gas industry.

Violation of the functioning of each of the following three segments of main gas pipelines "F" - "H" will lead to a significant gas deficit at consumers, up to $9 \%$ in total throughout the system, but the contribution to the synergetic effect from them will be lower than the disruption of the functioning of any segment of "I "-" J ". The failure of each of these five segments of main gas pipelines will lead to a significant gas deficit in consumers, $8-9 \%$ in total throughout the system. Therefore, segments of main gas pipelines "F" - "J" can also be attributed to critical objects of gas industry.

A similar situation can be traced with segments of main gas pipelines "O" - "R" and "S" - "T", the synergetic effect in case of a violation of any of these segments will be higher than violation of segments "K" "N" and "Q" - "R" respectively. It should be noted here that segments of main gas pipelines "N" - "T" are not critical objects, since the total gas deficit in the system in case of a violation of their functioning is $<5 \%$.

Violation of the functioning of several unconnected segments of main gas pipelines as well as the disruption of the functioning of the intersection of main gas pipelines are more likely to cause more harm to the system than the disruption of the functioning of one segment of main gas pipelines. Taking this fact into account, we can speak about the greater importance of segments of main gas pipelines as critical objects of gas industry with a large value of the criticality index.

\section{Conclusion}

The article shows the application of the method of determining critical elements in the networks of technical infrastructures when analysing the importance of the gas industry's critical objects. This method involves a systematic assessment of the consequences of failures of infrastructure elements to determine their criticality and is characterized by a sufficiently high computational resource capacity. It was originally used for the analysis of the electric power system, however, after appropriate adaptation, which consists in describing the response of the system to element failure, is suitable for studies of the vulnerability of gas supply systems. A detailed analysis of the results of the assessment of the importance of segments of main gas pipelines on two indicators is presented: the total gas deficit and the element criticality index. This indicator can be used to establish the order of priority of measures to increase the survivability of the Unified Gas Supply System of Russia.

Also, the suitability of the presented method in the studies of the gas sector in the part of determining segments of main gas pipelines with the greatest contribution to the possible synergetic effect in the case of disruption of the functioning of several segments was noted in the article. 
In the future, it is possible to apply the method of determining the critical elements in the networks of technical infrastructures to analyse the possible synergistic effect of the gas industry's critical objects.

\section{References}

1. E. Zio, Reliability Engineering \& System Safety, 94(2), pp.125-141. (2009).

2. G.E. Apostolakis, Risk analysis, 24(3), pp.515-520. (2004).

3. H. Liu, R.A. Davidson, T.V. Apanasovich, Reliability Engineering \& System Safety, 93(6), pp.897912. (2008).

4. L. Cuadra, S. Salcedo-Sanz, J. Del Ser, S. JimenezFernandez, Z.W. Geem, Energies, 8(9), pp.9211-9265. (2015).

5. M. Ouyang, Reliability engineering \& System safety, 121, pp.43-60. (2014).

6. S. Wang, L. Hong, X. Chen, Physica A: Statistical Mechanics and its applications, 391(11), pp.3323-3335. (2012).

7. J. Johansson, H. Hassel, Risk and Interdependencies in Critical Infrastructures: A Guideline for Analysis. London: Springer-Verlag, pp. 49-66. (2012).

8. S.M. Senderov, V.I. Rabchuk, A.V. Edelev, Izv. Ros. Akad. Nauk. Energetika - № 1. - p. 70-78. (2016).

9. H. Jonsson, J. Johansson, H. Johansson, Proceedings of the Institution of Mechanical Engineers, Part O: Journal of Risk and Reliability. Vol. 222. No. 2. P. 235-243. (2008).

10. J. Johansson, H. Hassel, E. Zio, Reliability Engineering \& System Safety. No. 120. P.27-38. (2013).

11. A.V. Edelev, S.M. Enikeeva, S.M. Senderov, Computational technologies. - Vol. 4, № 5. - p. 30 - 35 . (1999).

12. N.I. Voropai, S.M. Senderov, A.V. Edelev, Energy 42, pp. 3-9, (2012).

13. S.V. Vorobev, A.V. Edelev, Software products and systems. - №3. - p. 174 - 177. (2014).

14. L.R. Ford, D.R. Fulkerson. Flows in networks. Princeton University Press. 276p. (1966).

15. Export of the Russian Federation of the most important goods in 2011 - 2016 (according to the Federal Customs Service of Russia) http://customs.ru/ index.php?option=com _ newsfts\&view =category\&id= 52\&Itemid $=1978 \&$ limitstart $=60$.

16. InfoTEK Monthly oil and gas magazine.

№1, - P.154. (2017).

17. Ministry of Energy of the Russian Federation. Statistics. http://minenergo.gov.ru/activity/statistic. 